\title{
An appeal for reimbursement for innovative prevention measures in patient cardiovascular education
}

\section{Edward D Frohlich}

We are well aware that cardiovascular-related deaths and stroke are the most common cause of premature loss of life in the US and other industrialized societies. Fortunately, cardiovascular-related mortality has markedly diminished over recent years. Nevertheless, implementation of more vigorous preventive measures through direct patient education could reduce this morbidity and mortality even further. Personal education is required to reduce or cease intake of a well-known 'cast' of culprits: calories, alcohol, sodium and tobacco.

One look at the news media and it's obvious that obesity is rising. Overweight and obesity are prime risk factors for coronary heart disease, stroke, diabetes and end-stage renal disease, and the reality is that our children are prematurely and needlessly affected by these conditions. For years, the interested physician has referred selected patients to dieticians and nutritionists, but over recent years a greater proportion of our patients have become obese. Now, physicians must become actively involved in their patients' education. We need to develop office-based, physician-led risk-prevention programs and educate our patients.

For years, medical panels have advised that consuming more than $28 \mathrm{~g}$ ethanol daily increases arterial pressure in normotensive and hypertensive individuals, as well as being a source of calories. In addition, we know that excessive alcohol intake is responsible for adverse affects in patients with diabetes, hepatic and certain psychiatric disorders, and other diseases. Despite this knowledge, alcohol consumption in the US and other countries continues to rise.

Not only does sodium increase blood pressure, it also promotes structural and functional derangements in the heart, large arteries and kidneys. These functional derangements actively participate in cardiovascular and end-stage renal disease. Half of our sodium intake comes from salt surfeit in our diet, with the rest coming from sodium-based additives that preserve our
... personal

education

is required

to reduce or

cease intake

of a well-

known 'cast'

of culprits:

calories,

alcohol,

sodium and

tobacco.

ED Frohlich is the

Alton Ochsner

Distinguished Scientist at the Ochsner Clinic

Foundation, New

Orleans, LA, USA.

Competing interests

The author declared no

competing interests.

www.nature.com/clinicalpractice doi:10.1038/ncpcardio1183 canned, frozen and processed foods. Important measures have already been instituted by the FDA, limiting sodium content and making food labeling mandatory, but too frequently these measures go unheeded. With the ubiquity of sodium in most foods, the quantity is markedly increased pari passu with food quantity. Our regulatory and health groups urgently need to educate our patients, to reduce their daily sodium intake to no more that $2.3 \mathrm{~g}$.

We have already witnessed the benefits of reduction or cessation of tobacco consumption, but more action is necessary. While smoking has dramatically decreased in men, it continues to increase in teenagers and women. Only recently, we witnessed an increase in cardiovascularrelated death in women, while the same figure dropped in men. The media and medical authorities have attributed this rise to the increased prevalence of obesity, but cardiovascularrelated morbidity and mortality have diminished in the obese male population. I suggest that this increase in cardiovascular mortality may be related to the increased smoking rates in women. Penalties for smoking and measures to increase the cost of healthcare for smokers (either directly or through insurance) must be levied. These financial penalties could accrue to the benefit of further support of cardiovascular research and education.

Currently, there is only one disease for which patient education is compensable by the government-diabetes. Our professional societies and organizations must stand up and demand the same compensation for cardiovascular disease. This message is written with hope that the American Medical Association, American Heart and Stroke Association, and American Colleges of Physicians and Cardiology be heard with one strong voice to stimulate direct patient education by their physician members and staff. Direct patient education by the pharmaceutical industry has been dramatically effective; let this new compensable effort be supported by the patients' healthcare providers themselves! 